\title{
Beyond stability constraints: a biophysical model of enzyme evolution with selection for stability and activity
}

\author{
Julian Echave (jechave@unsam.edu.ar) \\ Escuela de Ciencia y Tecnología, Universidad Nacional de San Martín (UNSAM), \\ Martín de Irigoyen 3100, 1650 San Martín, Buenos Aires, Argentina
}

(Dated: August 23, 2018)

\begin{abstract}
Proteins trace trajectories in sequence space as their amino acids become substituted by other amino acids. The number of substitutions per unit time, the rate of evolution, varies among sites because of biophysical constraints. Several properties that characterize sites' local environments have been proposed as biophysical determinants of site-specific evolutionary rates. Thus, rate increases with increasing solvent exposure, increasing flexibility, and decreasing local packing density. For enzymes, rate increases also with increasing distance from the protein's active residues, presumably due to functional constraints. The dependence of rates on solvent accessibility, packing density, and flexibility has been mechanistically explained in terms of selection for stability. However, as I show here, a stability-based model fails to reproduce the observed rate-distance dependence, overestimating rates close to the active residues and underestimating rates of distant sites. Here, I pose a new biophysical model of enzyme evolution with selection for stability and activity $\left(M_{\mathrm{SA}}\right)$ and compare it with a stability-based counterpart $\left(M_{\mathrm{S}}\right)$. Testing these models on a structurally and functionally diverse dataset of monomeric enzymes, I found that $M_{\mathrm{SA}}$ fits observed rates better than $M_{\mathrm{S}}$ for most proteins. While both models reproduce the observed dependence of rates on solvent accessibility, packing, and flexibility, $M_{\mathrm{SA}}$ fits these dependencies somewhat better. Importantly, while $M_{\mathrm{S}}$ fails to reproduce the dependence of rates on distance from the active residues, $M_{\mathrm{SA}}$ accounts for the rate-distance dependence quantitatively. Thus, the variation of evolutionary rate among enzyme sites is mechanistically underpinned by natural selection for both stability and activity.
\end{abstract}

Keywords: Protein Evolution Model, Stability Constraints, Activity Constraints, Site-specific rates 


\section{INTRODUCTION}

From the early days of Molecular Evolution we know that some protein sites evolve more slowly than others. Ever since, we have interpreted such variation of rates among sites in terms of purifying selection to maintain function and structure: slowly evolving sites are those where mutations are more likely to be discarded by natural selection because they perturb the protein's structure or function too much [30, 47].

Further insight into the determinants of evolutionary rates came from quantitative studies of the dependence of rates on various metrics that describe the local micro-environment of protein sites. The two metrics that best correlate with rates are $R S A[10,16,17,48,50$, $61,62]$ and $W C N[16,35,52,54,61,62] . R S A$ is the Relative Solvent Accessibility, which measures solvent exposure; $W C N$ is the Weighted Contact Number, which measures local packing density. Beyond properties of the static native structure, some work has studied the dependence of rates on dynamical properties such the B-factor $(B)$ of X-ray experiments, which measures local flexibility [25, 32, 33, 36, 46, 53]. Evolutionary rate increases with increasing $R S A$ and $B$ and decreases with increasing $W C N$. These findings have led to the view that the rate of evolution is mainly determined by protein structure, increasing from a slowly evolving, buried, tightly packed, and rigid protein core, towards a rapidly evolving, solvent-exposed, loosely packed, flexible surface [15].

In the previous view, functional constraints play only a minor role, affecting the evolutionary conservation of just a few sites [15], such as the catalytic residues of enzymes and some of their immediate neighbors [3, 59]. However, such a short-range effect of active sites on evolutionary rates seems to be at odds with directed evolution experiments, in which often 2nd or 3rd shell mutations are needed to optimize an enzyme's function [9, 22]. Also, the very existence of allosteric enzymes, whose activities change due to coupling between distant sites, demonstrates the possibility of long-range couplings [7, 9]. Consistent with this, an early study of $\alpha / \beta$ - barrel enzymes found that evolutionary rate correlates not only with the structural trait $R S A$, but also with distance from the closest active residue $d_{\text {active }}[10]$. This finding was confirmed by a recent systematic study of a large and diverse dataset of enzymes that showed that, on average, site-specific substitution rates increase slowly with increasing $d_{\text {active }}$, which led to the conclusion that active sites influence evolutionary rates at long distances, affecting most protein sites [26]. 
Studying the dependence of evolutionary rate on metrics such as $R S A, W C N$, $B$, and $d_{\text {active }}$ is useful to develop phenomenological models which can be used for rate prediction. Moreover, such studies provide some insight into the possible biophysical forces that shape evolutionary sequence divergence. However, as good as they may be for predictive or heuristic purposes, phenomenological models have limited explanatory power. Explaining the origin of rate variation among sites and the relationship between rate and structural and dynamical metrics requires theoretical mechanistic models grounded on first principles.

Several theoretical biophysical models have been used to study evolutionary constraints on sequence divergence [13]. Most of these models assume that protein fitness depends only on protein stability. Such stability-based models account for much of the observed variation of evolutionary rates among sites [14, 24, 25, 27, 35]. Moreover, stability-based models reproduce quite well the dependence of site-specific rates on $R S A, W C N$, and $B[25,35]$. Thus these traits can largely be considered as proxies of protein stability, which would be the actual underlying cause of differential conservation of protein sites [15].

Even though selection for stability explains the dependence of rates on $R S A, W C N$, and $B$, it is unlikely to account for the observed rate- $d_{\text {active }}$ dependence, which is probably strongly influenced by selection on activity. The very few studies that consider selection on activity focus on ligand binding [13]. Ligand-binding constraints may lead to longrange epistasis [45], which is consistent with the rate- $d_{\text {active }}$ dependence found for enzymes. However, rather than ligand binding, the key to enzymatic activity is lowering the activation energy barrier of the catalyzed reaction. For a proper understanding of the dependence of site-specific evolutionary rate on $d_{\text {active}}$, we need biophysical models that include activity constraints based on realistic principles of enzymatic catalysis.

Here I propose a mechanistic biophysical stability-activity model of enzyme evolution, $M_{\mathrm{SA}}$, that includes selection for stability and activity explicitly. I compare this model with a stability model, $M_{\mathrm{S}}$, developed previously [14]. Using a diverse dataset of 160 monomeric enzymes, I assess whether $M_{\mathrm{S}}$ and/or $M_{\mathrm{SA}}$ account for the observed rate variation among sites and, especially, whether they explain the dependence of rate on the site-specific traits

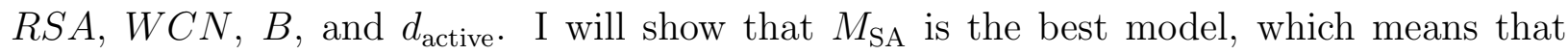
activity constraints influence the pattern of rate variation among sites. 


\section{NEW APPROACH}

I propose a new model of enzyme evolution, a stability-activity model, $M_{\mathrm{SA}}$, and, for the sake of comparison, I also consider a stability-based model $M_{\mathrm{S}} . M_{\mathrm{S}}$ and $M_{\mathrm{SA}}$ consider evolution as an origin-fixation process in which at each evolutionary time step a mutant is introduced by random mutation and is fixed, replacing its parent, with a certain fixation probability that accounts for natural selection. In general, any mutation may affect the enzyme's stability, measured by the change in free energy $\Delta \Delta G$, and its activity, which I measure by a change in activation free energy $\Delta \Delta G^{*}$. In $M_{\mathrm{S}}$ the fixation probability depends exclusively on $\Delta \Delta G$ and has a single parameter, $a_{\mathrm{S}}$, that represents selection pressure (Materials and Methods, Eq. 1). For $M_{\mathrm{SA}}$, the fixation probability depends on $\Delta \Delta G$ and on $\Delta \Delta G^{*}$, and on two selection-pressure parameters, $a_{\mathrm{S}}$ and $a_{\mathrm{A}}$ (Materials and Methods, Eq. 2).

To calculate $\Delta \Delta G$ and $\Delta \Delta G^{*}$, I used the Linearly Forced Elastic Network Model (LFENM), which represents a given protein as an elastic network of nodes (amino acids) connected by harmonic springs (interactions) and models mutations as random perturbations of the lengths of the springs that connect the mutated site to other sites [11, 12, 25, 35]. The mutational stability change, $\Delta \Delta G$, results from the mechanical stress introduced by the mutation minus the relaxation of part of this stress by modification of the mutant's conformation (Materials and Methods, Eq. 8). I calculated $\Delta \Delta G^{*}$ as the energy necessary to deform the mutant's catalytic residues so that they adopt their wild type conformation (Materials and Methods, Eq. 9).

Theoretical site-specific rates for model $M_{\mathrm{SA}}$ for a given protein are calculated as follows. First, an in silico mutational scan is performed introducing random mutations at each site and calculating $\Delta \Delta G$ and $\Delta \Delta G^{*}$. Then, site-specific rates are calculated using Eq. 12, Eq. 13 and Eq. 2. These depend on the model parameters $a_{\mathrm{S}}$ and $a_{\mathrm{A}}$, which represent the degree of selection for stability and activity, respectively, and are chosen to minimize the Mean Square Error between model rates $K_{M_{\mathrm{SA}}}$ and the empirical rates $K_{\mathrm{obs}}$. For model $M_{\mathrm{S}}$ a similar procedure is followed using only $\Delta \Delta G$ and optimizing $a_{\mathrm{S}}$ to minimize the MSE between $K_{M_{\mathrm{S}}}$ and $K_{\mathrm{obs}}$. All rates are relative to their protein average.

For a more detailed description of the models see Materials and Methods. 


\section{RESULTS}

I applied $M_{\mathrm{S}}$ and $M_{\mathrm{SA}}$ to a dataset of 160 monomeric enzymes with diverse sizes, functions, and structures. Since different proteins may be subject to different degrees of selection for stability and/or activity, I performed a protein by protein analysis. For each protein, I performed a mutational scan to calculate $\Delta \Delta G$ and $\Delta \Delta G^{*}$ for each of 19 point mutations at each protein site and I calculated the site-specific rates predicted by the models, $K_{M_{\mathrm{SA}}}$ and $K_{M_{\mathrm{S}}}$, by fitting the models' parameters to observed rate estimates, $K_{\mathrm{obs}}$, obtained from sequence alignments using the program rate4site [37]. See Supplementary Material file dataset.pdf for details on the proteins studied, their sizes, structural and functional classes, and the parameters of models $M_{\mathrm{SA}}$ and $M_{\mathrm{S}}$.

To test the models, I investigated two issues. First, which of the model rates, $K_{M_{\mathrm{SA}}}$ or $K_{M_{\mathrm{S}}}$, provides a better fit to empirical rates, $K_{\text {obs. }}$. Second, which model provides a better account of the the dependence of observed site-specific rates on the molecular traits $R S A$, $W C N, B$, and $d_{\text {active }}$.

\section{Fit between predicted and observed rates: $M_{\mathrm{SA}}$ outperforms $M_{\mathrm{S}}$}

So far, most biophysical studies have assumed that stability is the main, if not only, determinant of site-specific evolution, disregarding specific activity constraints altogether. Thus the first issue to consider is whether it is at all necessary to add selection for activity explicitly. In other words, are observed rates fit better by $M_{\mathrm{SA}}$ than by $M_{\mathrm{S}}$ ?

Figure 1 shows empirical rates vs. model predictions for protein 1PMI, which I use as an example. (1PMI is the pdb code of the Phosphomannose isomerase of Candida albicans.) Typically, models are compared using $R^{2}$, the square Pearson correlation coefficient between predicted and observed rates. For $1 \mathrm{PMI}, R^{2}\left(K_{M_{\mathrm{SA}}}, K_{\mathrm{obs}}\right)=0.57>R^{2}\left(K_{M_{\mathrm{SA}}}, K_{\mathrm{obs}}\right)=0.41$. Thus, in this case adding activity constraints increases the explained variance by $16 \%$. I consider $R^{2}$ for the sake of comparison with related work and to have an idea of the magnitude of improvement that results from adding activity constraints. However, for nonlinear models, the Akaike Information Criterion $(A I C)$ is better for model selection[55]. The best model is that with lowest $A I C$. For $1 \mathrm{PMI}, \triangle A I C=A I C\left(M_{\mathrm{SA}}\right)-A I C\left(M_{\mathrm{S}}\right)=-127.4$, thus $M_{\mathrm{SA}}$ should be selected. Using $\triangle A I C$, we can calculate Akaike Weights, $w\left(M_{\mathrm{SA}}\right)$ and 
$w\left(M_{\mathrm{S}}\right)$, which give the weight of evidence for each model. For $1 \mathrm{PMI}, w\left(M_{\mathrm{SA}}\right)=1$ and $w\left(M_{\mathrm{S}}\right)=0$. To summarize, for $1 \mathrm{PMI}$, rates predicted with $M_{\mathrm{SA}}$ fit observed rates better than rates predicted by $M_{\mathrm{S}}$.

I repeated the previous analysis for each of the enzymes of the dataset. Figure 2 shows that $M_{\mathrm{SA}}$ outperforms $M_{\mathrm{S}}$ for most proteins: $M_{\mathrm{SA}}$ has the lowest AIC for $131 / 160$ proteins (Binomial test: $\left.p=82 \%, P=6 \times 10^{-17}\right)$. Also, $R^{2}(M S A)>R^{2}(M S):<\Delta R^{2}>=<$ $R^{2}(M S A)-R^{2}(M S)>=0.042 \pm 0.06$ ( \pm two standard deviations) ( $\mathrm{t}$ Test: $t=12.9, P=0$ ). Thus, adding activity constraints results in better predictions, which means that activity constraints have observable effects on the patterns of rate variation among sites for most proteins.

\section{Dependence of site-specific rates on molecular traits: MSA outperforms MS}

Beyond the overall goodness of fit, studied in the previous section, a good model should also reproduce how site-specific rates depend on the traits with which they correlate: $R S A$, $W C N, R S A$, and $d_{\text {active }}$, which I will refer to as molecular traits or empirical predictors. In this section, I explore whether the models account for the dependence of observed rates on these empirical predictors.

Following the scheme of the previous section, I start with the example protein 1PMI. Figure 3 shows observed and model substitution rates vs. the various empirical predictors. For a given $K$ (observed or model rate) vs. $X$ (empirical predictor), let $\widehat{K}(X)$ be the smooth fit to the scatter plot obtained using local polynomial regression (shown with lines in Figure 3, see Materials and Methods for details). Let $\operatorname{RMSE}(M, X)$ be the Root Mean Square Error between smooth trends $\widehat{K}_{M}(X)$ and $\widehat{K}_{\text {observed }}(X)$, and let $\triangle R M S E(X)=$ $R M S E\left(M_{\mathrm{SA}}, X\right)-R M S E\left(M_{\mathrm{S}}, X\right)$. The model that has lowest $R M S E(X)$ reproduces

better the $\widehat{K}_{\text {observed }}(X)$ trend. For 1PMI, $\triangle R M S E(R S A)=0.00, \triangle R M S E(W C N)=$ -0.08, $\triangle R M S E(B)=-0.06$, and $\triangle R M S E\left(d_{\text {active }}\right)=-0.22$. Thus, in this case, both models reproduce dependence of rates on $R S A$ similarly, $M_{\mathrm{SA}}$ provides a better fit for the the dependence of rates on $W C N$ and $B$, and $M_{\mathrm{SA}}$ provides a much better account of the dependence of rates on $d_{\text {active }}$. From Figure 3 we see that relative to $M_{\mathrm{SA}}, M_{\mathrm{S}}$ overestimates (underestimates) rates at small (large) $d_{\text {active, }}$ small (large) $B$ and large (small) $W C N$.

In addition to smooth trends, Figure 3 shows the rates for each site. We see that $M_{\mathrm{SA}}$ 
reproduces better than $M_{\mathrm{S}}$ not only the average trend, but also the dispersion of rates around the trend for $R S A, W C N$, and $B: K_{M_{\mathrm{S}}}$ is much less variable around the smooth trend than $K_{M_{\mathrm{SA}}}$ and $K_{\mathrm{obs}}$. The reason for this is that activity constraints, which are not accounted for by $M_{\mathrm{S}}$, contribute to this dispersion. For instance, active residues and their immediate neighbors deviate strongly from the smooth trends $\widehat{K}(R S A), \widehat{K}(W C N)$, and $\widehat{K}(B)$ for both $K_{M_{\mathrm{SA}}}$ and $K_{\mathrm{obs}}$, but not for $K_{M_{\mathrm{S}}}$. Thus, including activity constraints affects the rate-trait dependence not only for $d_{\text {active, }}$ where it was to be expected, but for all four of the molecular traits.

To test whether $M_{\mathrm{SA}}$ reproduces rate-predictor trends better than $M_{\mathrm{S}}$ for other proteins as well, I repeated the previous analysis on each of the 160 enzymes of the dataset. Figure 4 shows $R M S E\left(M_{\mathrm{SA}}\right)$ vs $R M S E\left(M_{\mathrm{S}}\right)$ for all proteins. Points below the diagonal represent cases for which $M_{\mathrm{SA}}$ reproduces trends better than $M_{\mathrm{S}}$. Counting cases for which $\triangle R M S E=R M S E\left(M_{\mathrm{SA}}\right)-R M S E\left(M_{\mathrm{S}}\right)<0$, we see that $M_{\mathrm{SA}}$ reproduces better than $M_{\mathrm{S}}$ the dependence of site-specific rates with $d_{\text {active }}, B$, and $W C N$, while it preforms similarly for the rate- $R S A$ dependence (Table 1 ). Taking into account the magnitude of the improvements, as shown in Table 2, the average error difference $\langle\triangle R M S E\rangle$ is negative for all four traits; $M_{\mathrm{SA}}$ fits rate-predictor trends better for all traits, with the largest improvement for $d_{\text {active }}$ and the smallest for $R S A$.

To summarize, $M_{\mathrm{S}}$ fails to reproduce the observed rate- $d_{\text {active }}$ dependence (it overestimates rates of sites close to the active site and overestimates rates of distant sites), whereas $M_{\mathrm{SA}}$ accounts well for this dependence. Even though both models give good descriptions of the rate-trait dependence for $R S A, W C N$, and $B, M_{\mathrm{SA}}$ fits these trends better.

\section{DISCUSSION}

I have posed two models of enzyme evolution, a model with selection for stability, $M_{\mathrm{S}}$, and a model with selection for stability and activity $M_{\mathrm{SA}}$. That selection acts on stability is well established and is the key assumption of the vast majority of biophysical models of protein evolution proposed so far [13]. However, natural selection acts not only on protein stability but on enzyme activity parameters such as $k_{\text {cat }} / K_{M}[1,2]$. Further, activationenergy factors are needed to explain, for instance, the effect of temperature on the growth rate, thus fitness, of viruses and bacteria [8, 20, 21]. Moreover, in some cases selection 
on activity is stronger than selection on stability [31]. Despite the extensive evidence of selection on protein activity, there is a general lack of biophysical models of evolution that consider activity constraints explicitly [13]. The $M_{\mathrm{SA}}$ model proposed here is a step towards filling this gap.

The key to $M_{\mathrm{S}}$, which was derived in detail previously [6, 14], is a fixation probability function that depends only on mutational stability changes, $\Delta \Delta G$ (Eq. 1). In contrast, the fixation probability of $M_{\mathrm{SA}}$ depends not only on $\Delta \Delta G$, but also on mutational changes of the activation free energy of the enzyme-catalyzed reaction, $\Delta \Delta G^{*}$. In [14], $\Delta \Delta G$ was calculated using the all-atom empirical energy function of the program FoldX. Here, I calculated $\Delta \Delta G$ (and $\left.\Delta \Delta G^{*}\right)$ using a simpler coarse-grained Linearly-Forced Elastic Network Model (LFENM) Despite its simplicity, LFENM predicts $\Delta \Delta G$ values with similar properties to those obtained using FoldX. (For instance, the average $\Delta \Delta G$ increases with the number of contacts of a site. I will present these results in a separate publication.) More importantly, the models are validated by their success in fitting the empirical site-specific substitution rates and capturing the dependence of rates on several molecular traits.

Even if both $M_{\mathrm{S}}$ and $M_{\mathrm{SA}}$ predict site-specific evolutionary rates in good agreement with observations, I have found that $M_{\mathrm{SA}}$ fits observed empirical rates better. This means that evolutionary rate variation among sites in enzymes is determined not only but selection on thermodynamic stability, but also by selection on specific enzymatic activity.

The most noticeable difference between the two models is that $M_{\mathrm{SA}}$ reproduces correctly the dependence of site-specific rates on distance from the active site, $d_{\text {active }}$, which $M_{\mathrm{S}}$ cannot explain. Thus, $M_{\mathrm{SA}}$ provides the mechanistic biophysical underpinning for the long-range influence of active sites on the evolution of other sites observed in phenomenological data analysis studies [10, 26].

Surprisingly, I found that activity constraints affect the dependence of rates on other molecular traits as well. $M_{\mathrm{S}}$ reproduces quite well the dependence of rates on $R S A, W C N$, and $B$, consistently with previous work that explained this dependence in terms of selection for stability $[13,15,25,27,35]$. However, $M_{\mathrm{SA}}$ fits the dependence of rates on these traits even better. Specifically, $M_{\mathrm{S}}$ tends to overestimate rates in regions of high $W C N$ and low $B$. The reason is simple: active sites tend to be in these rigid (low $B$ ) highly-packed (large $W C N$ ) buried (low $R S A$ ) regions, where stability constraints are high, inducing extra activity constraints. 
To summarize, I posed the model $M_{\mathrm{SA}}$, a new biophysical model of enzyme evolution that considers explicitly selection for stability and activity, and I compared $M_{\mathrm{SA}}$ with a stability-based model $M_{\mathrm{S}}$ on a diverse dataset of monomeric enzymes. I found that sitespecific substitution rates predicted by the model $M_{\mathrm{SA}}$ fit observed rates better than rates predicted by $M_{\mathrm{S}}$. Moreover, the dependence of observed rates on the molecular traits $R S A$, $B, W C N$, and $d_{\text {active }}$ is better accounted for by $M_{\mathrm{SA}}$ than by $M_{\mathrm{S}}$. Thus, activity constraints have a significant effect on patterns of rate variation among sites and this is adequately modeled by the stability-activity model proposed here.

I believe the $M_{\mathrm{SA}}$ model will be helpful to explore several fundamental issues and to develop applications. For example, $M_{\mathrm{SA}}$ might be helpful to explain why proteins evolve to be moderately efficient $[1,2]$, just as stability-based models have explained why proteins are marginally stable $[23,57]$. Another classical issue that may be explored using $M_{\mathrm{SA}}$ is the evolutionary implications of a trade-off between stability and activity [42]. On the applied side, $M_{\mathrm{SA}}$ could be used to improve active-site prediction [26]. Another application would be to add activity constraints, as modeled by $M_{\mathrm{SA}}$, to improve probabilistic evolution models used for phylogenetic inference purposes [49]. In general, I expect that developing biophysical models of protein evolution that consider selection for stability and activity, such as $M_{\mathrm{SA}}$, is a promising research endeavor that will advance our understanding of protein evolution and impact many areas of evolutionary biology.

\section{MATERIALS AND METHODS}

\section{Two models of enzyme evolution}

\section{Origin-fixation models}

When mutations are rare, evolution can be thought of as an origin-fixation process [38]. Most of the time the population consists of a single genotype, which is the current wild type. When a new mutant originates, it competes with the wild type and is destined to become either lost or fixed. For such an origin-fixation process, evolutionary dynamics is determined by the fixation probability, which is the probability that the mutant prevails, replacing the previous wild type. The two models of evolution described below are origin-fixation models. 
The stability model $\left(M_{S}\right)$

I consider a stability-based model, $M_{\mathrm{S}}$, that has been used to predict site-specific rates of evolution [14]. $M_{\mathrm{S}}$ is an origin-fixation model with fixation probability:

$$
p_{M_{\mathrm{S}}}^{\mathrm{fix}}= \begin{cases}1 & \text { for } \Delta \Delta G<0 \\ e^{-a_{\mathrm{S}} \Delta \Delta G} & \text { for } \Delta \Delta G>=0\end{cases}
$$

where $\Delta \Delta G=\Delta G_{\mathrm{mut}}-\Delta G_{\mathrm{wt}}$ is the stability change due to the mutation. ( $\Delta G_{\mathrm{mut}}$ and $\Delta G_{\mathrm{wt}}$ are the folding stabilities of mutant and wild type, respectively). The parameter $a_{\mathrm{S}} \geq 0$ represents the degree of selection pressure for protein stability.

\section{The stability-activity model $\left(M_{S A}\right)$}

The purpose of this work is to go beyond stability constraints and study the effects of specific functional constraints on patterns of rate variation among sites. To this end, I pose a stability-activity model of enzyme evolution, $M_{\mathrm{SA}}$, with selection depending not only on stability but also on enzyme activity. $M_{\mathrm{SA}}$ is specified by the following fixation probability:

$$
p_{M_{\mathrm{SA}}}^{\mathrm{fix}}= \begin{cases}1 & \text { for } d x>0 \\ e^{d x} & \text { for } d x<=0\end{cases}
$$

with

$$
d x=-a_{\mathrm{S}} \Delta \Delta G-a_{\mathrm{A}} \Delta \Delta G^{*}
$$

where $\Delta \Delta G=\Delta G_{\mathrm{mut}}-\Delta G_{\mathrm{wt}}$ is the mutational change of stability and $\Delta \Delta G^{*}=\Delta G_{\mathrm{mut}}^{*}$ $\Delta G_{\mathrm{wt}}^{*}$ is the mutational change of the activation energy of the reaction catalyzed by the enzyme. The model has two positive parameters, $a_{\mathrm{S}}$ and $a_{\mathrm{A}}$, that represent the degree of selection pressure for stability and activity, respectively.

\section{Calculation of $\Delta \Delta G$ and $\Delta \Delta G^{*}$ using the Linearly Forced Elastic Network Model}

To apply the models proposed above, we need to calculate mutational changes of stability, $\Delta \Delta G$, and of activation energy, $\Delta \Delta G^{*}$. To this end, I use the Linearly Forced Elastic Network Model (LFENM) [11, 12, 25, 35]. 


\section{Proteins are modeled as Elastic Networks}

The LFENM models proteins as networks of nodes, representing amino acids, connected by harmonic springs, representing interactions. The energy function of a given wild-type protein is given by:

$$
V_{\mathrm{wt}}=\frac{1}{2} \sum_{i<j} k_{i j}\left(d_{i j}-l_{i j}\right)^{2}
$$

where $k_{i j}$ and $l_{i j}$ are, respectively, the force constant and equilibrium length of the spring connecting nodes $i$ and $j$. Various Elastic Network Models have been developed over the years [4, 18, 34]. Here, I used a model proposed by Ming and Wall [43]: amino acids are

represented by single nodes; nodes are connected if they are within $R_{0}=10.5 \AA ; l_{i j}$ is the distance between the nodes in the PDB structure; the force constants are $k_{i j}=189 \mathrm{Kcal} / \mathrm{Mol}$ for sequence neighbors and $k_{i j}=4.5 \mathrm{Kcal} / \mathrm{Mol}$ otherwise. Since for evolutionary models it is better to represent residues using side chains rather than $C_{\alpha}$ [35], I placed nodes at the side-chain geometric centers (except for glycine, which has no side chain, for which I used $C_{\alpha}$ coordinates).

\section{Mutations are modeled as perturbations}

The LFENM models mutations as perturbations of the equilibrium lengths of the springs that connect the mutated site to its neighbors. Thus, introducing a mutation at site $r$ results in a mutant with potential energy:

$$
V_{\mathrm{mut}}=\frac{1}{2} \sum_{i<j} k_{i j}\left(d_{i j}-l_{i j}-\delta l_{i j}\right)^{2}
$$

where $\delta l_{i j}$ is a random perturbation added to $l_{i j}$. Only contacts of site $r$ are perturbed (i.e. $\delta l i j \neq 0$ only for $i=r$ or $j=r$, and $\delta l_{i j}=0$ otherwise). I picked the non-zero $\delta l_{i j}$ for the perturbed contacts independently from a normal distribution $N(\mu=0, \sigma=0.3 \AA)$.

Mutations distort the native conformation

$V_{\text {mut }}$ is minimum at the mutant's equilibrium conformation, which is given by:

$$
\mathbf{r}_{\mathrm{mut}}^{0}=\mathbf{r}_{\mathrm{wt}}^{0}+\delta \mathbf{r}^{0}
$$


where $\mathbf{r}_{\mathrm{wt}}^{0}$ and $\mathbf{r}_{\mathrm{mut}}^{0}$ are the column vectors of node coordinates that represent the native conformations of the wild type and the mutant, respectively. The mutational conformational change can be calculated using:

$$
\delta \mathbf{r}^{0}=-\mathbf{K}^{-1} \mathbf{f}
$$

where $\mathbf{K}$ is the Hessian matrix of $V_{\mathrm{wt}}$ and $V_{\text {mut }}$. (Since $\mathbf{K}$ is not affected by linear perturbations, $\mathbf{K}_{\mathrm{mut}}=\mathbf{K}_{\mathrm{wt}}=\mathbf{K}$.) The vector $\mathbf{f}$ is a "force" that models the mutation and is calculated from the perturbations $\delta l_{i j}$. For more details see $[11,12]$.

\section{$\Delta \Delta G$ and $\Delta \Delta G^{*}$ are mechanical deformation energies}

Using Eq. 4 and Eq. 5, assuming no variation of the unfolded state free energy, expanding potentials up to second order, and using basic statistical thermodynamics, it is possible to derive:

$$
\Delta \Delta G=\frac{1}{2} \sum_{j \sim i} k_{i j} \delta l_{i j}^{2}-\frac{1}{2}\left(\delta \mathbf{r}^{0}\right)^{T} \mathbf{K} \delta \mathbf{r}^{0},
$$

where $\Delta \Delta G$ is the stability change due to the mutation, $\delta \mathbf{r}^{0}$ is the conformational change, calculated using Eq. 7, and $T$ stands for transpose. The first term of Eq. 8 is always positive and represents the total stress introduced by the mutation [25, 35]; the second term is negative and represents the distortion of the mutant to relax part of the stress.

To calculate the change in activation free energy $\Delta \Delta G^{*}$, I assume that the activation free energy is a sum of two terms a distortion energy plus a vertical binding energy [51, 56]. The distortion energy is the energy needed to change the conformation of enzyme and substrate from their free forms to their "poses" in the activated complex. The vertical binding energy is the free energy released when enzyme and substrate in the right poses bind together. I further assume that mutations at non-catalytic residues modify activity by distorting the active site's structure, which modifies the distortion energy. Then, $\Delta \Delta G^{*}$ equals the energy necessary to deform the mutant's catalytic residues so that they adopt their wild-type conformation, which can be derived from Eq. $5 \Delta \Delta G^{*}$ to be given by:

$$
\Delta \Delta G^{*}=\frac{1}{2}\left(\delta \mathbf{r}_{a}^{0}\right)^{T} \mathbf{K}_{a a}^{\mathrm{eff}} \delta \mathbf{r}_{a}^{0}
$$

where $\delta \mathbf{r}_{a}^{0}$ is the conformational change of the active residues due to the mutation and

$$
\mathbf{K}_{a a}^{\mathrm{eff}}=\mathbf{K}_{a a}-\mathbf{K}_{a n} \mathbf{K}_{n n}^{-1} \mathbf{K}_{n a}
$$


is a matrix that governs effective energies of deformation within the space defined by the coordinates of the active residues, taking into account their coupling with the rest of the enzyme. (To derive Eq. 10, the Hessian $\mathbf{K}$ was divided in four blocks:

$$
\mathbf{K}=\left[\begin{array}{ll}
\mathbf{K}_{a a} & \mathbf{K}_{a n} \\
\mathbf{K}_{n a} & \mathbf{K}_{n n}
\end{array}\right]
$$

where the diagonal blocks $\mathbf{K}_{a a}$ and $\mathbf{K}_{n n}$ correspond to the active $(a)$ and non-active $(n)$ residues, respectively, and the off-diagonal blocks $\mathbf{K}_{a n}$ and $\mathbf{K}_{n a}$ couple active and non-active residues. See [41] for the derivation of an equation similar to Eq. 10.)

A note of caution is in order. Since the perturbation term of the LFENM is linear, the hessian $\mathbf{K}$ is not affected by the mutation, thus $\Delta \Delta G$ (Eq. 8) and $\Delta \Delta G^{*}$ (Eq. 9) do not include entropic changes that may have an effect on catalysis in the absence of conformational changes $[39,40]$.

\section{Testing models against data}

Dataset

I tested the models on 160 of the 524 enzymes used in [26]. Specifically, I kept only monomeric enzymes. I further removed those enzymes that had missing amino acids or broken chains, which could result in wrong elastic network models. The dataset contains representatives of all main SCOP structural classes [44] and the six main EC functional classes [60]. No two enzymes of the dataset have more than $25 \%$ sequence identity. Catalytic residue information was obtained from the Catalytic Site Atlas [19]. PDB structures of these proteins were obtained from the RCSB protein database[5]. The list of proteins,their structural and functional classes, size and number of domains, and model parameters can be found in Supplementary Material file dataset.pdf.

\section{Assessing whether models fit observed rates}

First, I compared model rates with observed rates. Let $K_{\text {obs }}$ denote the site-specific substitution rates estimated from multiple sequence alignments, which I call "observed" or "empirical" rates. I normalized these rates so that their average over sites is $\left\langle K_{\text {obs }}\right\rangle=1$ : 
$K_{\text {obs }}$ are rates relative to the protein mean. Briefly, calculating $K_{\text {obs }}$ involves finding homologous sequences, aligning them, inferring a phylogeny, and using the sequence alignment and phylogeny as input for the program rate4site to estimate the substitution rate of each site [37]. Here I have used the site-specific rates calculated by Jack et al. [26], where the reader is referred to for further details.

Let $K_{M}$ denote the site-specific rates predicted by model $M$, where $M$ is either $M_{\mathrm{S}}$ or $M_{\mathrm{SA}}$. Let $\mathbf{a}$ be the list of parameters: $\mathbf{a}=a_{\mathrm{S}}$ for $M_{\mathrm{S}}$ and $\mathbf{a}=\left(a_{\mathrm{S}}, a_{\mathrm{A}}\right)$ for $M_{\mathrm{SA}}$. The relative substitution rate of site $r$ is given by:

$$
K_{M}^{r}(\mathbf{a})=\alpha \omega_{M}(\mathbf{a})^{r}
$$

with

$$
\omega_{M}^{r}(\mathbf{a})=\left\langle p_{M}^{\mathrm{fix}}(\mathbf{a})\right\rangle^{r}
$$

where $p_{M}^{\mathrm{fix}}$ is the model's fixation probability (Eq. 1 or Eq. 2), $\langle\ldots\rangle^{r}$ stands for averaging over all possible point mutations at site $r$, and $\alpha$ is a constant that normalizes rates so that the average over sites is $\left\langle K_{M}\right\rangle=1$.

To calculate model rates for a given protein, I started by performing a full mutational scan: at each site, I introduced $N=19$ simulated mutations and calculated, for each mutation, $\Delta \Delta G$ (Eq. 8) and $\Delta \Delta G^{*}(\mathrm{Eq} 9)$. Then, for $M=M_{\mathrm{S}}$ and $M=M_{\mathrm{SA}}$, I determined the optimal parameter values minimizing the residual sum of squares $R S S=\sum_{r}\left(K_{\mathrm{obs}}^{r}-K_{M}^{r}(\mathbf{a})\right)^{2}$ using the general purpose optimization function optim of $\mathrm{R}$ package stats.

To assess how well $M_{\mathrm{S}}$ and $M_{\mathrm{SA}}$ predictions fit the data, I compared model rates $K_{M_{\mathrm{S}}}$ and $K_{M_{\mathrm{SA}}}$ with observed rates $K_{\mathrm{obs}}$ for each protein. The most frequently used goodnessof-fit measure is the square Pearson correlation coefficient between model predictions and observations $R^{2}$. Here, I used $R^{2}$ to have an idea of the degree of improvement provided by $M_{\mathrm{SA}}$ over $M_{\mathrm{S}}$ (i.e. how much more of the variance of $K_{\mathrm{obs}}$ does $M_{\mathrm{SA}}$ account for). However, for non-linear models such as $M_{\mathrm{S}}$ and $M_{\mathrm{SA}}, R^{2}$ is not adequate for model selection [55]. Thus, for model selection, I used the Akaike Information Criterion $(A I C)$ and the associated Akaike weights $w_{A I C}$ [55]. Specifically, for each model $M$, I calculated $A I C=2 k-2 \ln L$, where $L$ is the maximum likelihood and $k$ is the number of parameters. $A I C$ is smaller for larger likelihoods and less parameters; the best model is that with the smallest $A I C$. Further, for each model $M$, I calculated the Akaike weight $w_{A I C}(M)$, which gives the weight of evidence for $M$, using $w_{A I C}(M)=e^{-0.5 A I C(M)} / \sum_{M} e^{-0.5 A I C(M)}$. 


\section{Assessing whether models account for the dependence of rates on empirical predictors}

In addition to assessing the $K_{M}$ vs. $K_{\text {obs }}$ goodness of fit, I studied whether the models are able to reproduce correctly the dependence of site-specific rates on molecular traits that characterize the local micro-environment of protein sites. Specifically, I considered four molecular traits that have been used as empirical predictors of site-specific rates: $R S A$, $W C N, B$, and $d_{\text {active }}$, defined next.

- $R S A^{r}$ is the relative solvent accessibility of site $r$. It measures the fraction of the site's residue exposed to the solvent and is calculated as follows. First, the accessible surface area (ASA) is calculated using the software mkdssp [28, 29]. Then, ASAs are normalized by diving by the maximum solvent accessibility for each residue in a Gly$\mathrm{X}$-Gly tripeptide [58]. $R S A=0$ is assigned to peptide linkages across chains, typically disulfide bridges. Here I used the $R S A$ values of [26]. In principle, $R S A$ should vary from $R S A=0$ for completely buried sites to $R S A=1$ for completely exposed sites. Sometimes, the calculation protocol results in $R S A>1$, which is unphysical. In such cases, I set $R S A=1$.

- $W C N^{r}$ is the weighted contact number of site $r$, defined by:

$$
W C N^{r}=\sum_{q \neq r} \frac{1}{r_{r q}^{2}},
$$

where, $r_{r q}$ is the distance between the geometric centers of the side-chain atoms of residues $r$ and $q$, respectively [35]. For glycine, which has no side chain, I used $C_{\alpha}$ coordinates.

- $B$ is a measure that quantifies the local flexibility of a site. I calculated $B$ using the B-factors available in the protein's pdb file. The B-factor of an atom is proportional to its Mean Square Fluctuation (MSF) with respect to its coordinates in the native conformation. Here, I defined $B$, which I refer to as a residue B-factor, as the average of the B-factors of all heavy atoms of the residue's side chain. For glycine, I used the B-factor of its $\alpha$ carbon instead.

- $d_{\text {active }}^{r}$ is the distance from residue $r$ to the nearest catalytic residue. As for $W C N$, I considered distances between side-chain geometric centers ( $C_{\alpha}$ for glycines). By definition, a residue is a catalytic residue if and only if $d_{\text {active }}=0$. 
To describe the dependence of site-specific rate $K$ on site-specific molecular trait $X, \mathrm{I}$ obtained smooth fits to the $K$ vs. $X$ scatterplots, using local polynomial regression using the function loess of $\mathrm{R}$ package stats. Specifically, for each protein, I calculated smooth functions $\widehat{K}(X)$ for $K=K_{\mathrm{obs}}, K_{M_{\mathrm{S}}}, K_{M_{\mathrm{SA}}}$ and $X=R S A, W C N, B, d_{\text {active }}$. Then, I quantified how well $M$ accounts for the $K_{\mathrm{obs}} X$ dependence using the Root Mean Square Error: $\operatorname{RMSE}(M, X)=\sum_{i}\left[\widehat{K}_{\text {observed }}\left(X_{i}\right)-\widehat{K}_{M}\left(X_{i}\right)\right]^{2}$, where $X_{i}$ are evenly spaced points that cover the range of $X$. The model with lowest $\operatorname{RMSE}(M, X)$ is the one that best describes the $K_{\text {obs }} \sim X$ dependence; $\triangle R M S E(X) \equiv R M S E\left(M_{\mathrm{SA}}, X\right)-R M S E\left(M_{\mathrm{S}}, X\right)$ is negative (positive) if $M_{\mathrm{SA}}\left(M_{\mathrm{S}}\right)$ provides the best fit to the dependence of $K_{\text {obs }}$ on trait $X$.

\section{ACKNOWLEDGMENTS}

Most of this work was done at the Max Planck Institute for the Physics of Complex Systems (Dresden, Germany), where I was on sabbatical. This work was supported by Consejo Nacional de Investigaciones Científicas y Técnicas (grant number PIP 112201501 00385 CO) and by Agencia Nacional de Promoción Científica y Tecnológica (grant number PICT-2016-4209).

\section{SUPPLEMENTARY MATERIAL}

File dataset.pdf contains further information on the dataset.

[1] Bar-Even, A., Noor, E., Savir, Y., Liebermeister, W., Davidi, D., Tawfik, D. S., and Milo, R. 2011. The moderately efficient enzyme: Evolutionary and physicochemical trends shaping enzyme parameters. Biochemistry, 50: 4402-4410.

[2] Bar-Even, A., Milo, R., Noor, E., and Tawfik, D. S. 2015. The moderately efficient enzyme: futile encounters and enzyme floppiness. Biochemistry, 54: 4969-4977.

[3] Bartlett, G. J., Porter, C. T., Borkakoti, N., and Thornton, J. M. 2002. Analysis of catalytic residues in enzyme active sites. J. Mol. Biol., 324: 105-121.

[4] Bastolla, U. 2014. Computing protein dynamics from protein structure with elastic network models. WIREs Comput. Mol. Sci., 4: 488-503. 
[5] Berman, H. M., Westbrook, J., Feng, Z., Gilliland, G., Bhat, T. N., Weissig, H., Shindyalov, I. N., and Bourne, P. E. 2000. The Protein Data Bank. Nucleic Acids Res., 28: 235-242.

[6] Bloom, J. D. and Glassman, M. J. 2009. Inferring Stabilizing Mutations from Protein Phylogenies: Application to Influenza Hemagglutinin. PLoS Comput. Biol., 5: e1000349.

[7] Changeux, J.-P. P. 2013. 50 years of allosteric interactions: The twists and turns of the models. Nat. Rev. Mol. Cell Biol., 14: 819-829.

[8] Chen, P. and Shakhnovich, E. I. 2010. Thermal adaptation of viruses and bacteria. Biophys. J., 98: 1109-1118.

[9] Currin, A., Swainston, N., Day, P. J., and Kell, D. B. 2015. Synthetic biology for the directed evolution of protein biocatalysts: Navigating sequence space intelligently. Chem. Soc. Rev., 44: $1172-1239$.

[10] Dean, A. M., Neuhauser, C., Grenier, E., and Golding, G. B. 2002. The pattern of amino acid replacements in alpha/beta-barrels. Mol. Biol. Evol., 19: 1846-1864.

[11] Echave, J. 2008. Evolutionary divergence of protein structure: The linearly forced elastic network model. Chem. Phys. Lett., 457: 413-416.

[12] Echave, J. and Fernández, F. M. 2010. A perturbative view of protein structural variation. Proteins Struct. Funct. Bioinforma., 78: 173-180.

[13] Echave, J. and Wilke, C. 2017. Biophysical models of protein evolution: Understanding the patterns of evolutionary sequence divergence. Annu. Rev. Biophys., 46: 85-103.

[14] Echave, J., Jackson, E. L. E., and Wilke, C. O. C. 2015. Relationship between protein thermodynamic constraints and variation of evolutionary rates among sites. Phys. Biol., 12: 025002.

[15] Echave, J., Spielman, S. J., and Wilke, C. O. 2016. Causes of evolutionary rate variation among protein sites. Nat. Rev. Genet., 17: 109-121.

[16] Franzosa, E. A. and Xia, Y. 2009. Structural determinants of protein evolution are contextsensitive at the residue level. Mol. Biol. Evol., 26: 2387-2395.

[17] Franzosa, E. A. and Xia, Y. 2012. Independent effects of protein core size and expression on residue-level structure-evolution relationships. PLoS One, 7: e46602.

[18] Fugebakk, E., Reuter, N., and Hinsen, K. 2013. Evaluation of Protein Elastic Network Models Based on an Analysis of Collective Motions. J. Chem. Theory Comput., 9: 5618-5628. 
[19] Furnham, N., Holliday, G. L., de Beer, T. A. P., Jacobsen, J. O. B., Pearson, W. R., and Thornton, J. M. 2014. The Catalytic Site Atlas 2.0: cataloging catalytic sites and residues identified in enzymes. Nucleic Acids Res., pages D485-D489.

[20] Ghosh, K. and Dill, K. 2010. Cellular proteomes have broad distributions of protein stability. Biophys. J., 99: 3996-4002.

[21] Ghosh, K., De Graff, A. M. R., Sawle, L., and Dill, K. A. 2016. Role of protein physical chemistry in cell behavior. J. Phys. Chem. B, 120: 9549-9563.

[22] Goldsmith, M. and Tawfik, D. S. 2017. Enzyme engineering: Reaching the maximal catalytic efficiency peak. Curr. Opin. Struct. Biol., 47: 140-150.

[23] Goldstein, R. a. 2011. The evolution and evolutionary consequences of marginal thermostability in proteins. Proteins Struct. Funct. Bioinforma., 79: 1396-1407.

[24] Goldstein, R. A. and Pollock, D. D. 2017. Sequence entropy of folding and the absolute rate of amino acid substitutions. Nat. Ecol. Evol., 1: 1923-1930.

[25] Huang, T.-T., Del Valle Marcos, M. L., Hwang, J.-K., and Echave, J. 2014. A mechanistic stress model of protein evolution accounts for site-specific evolutionary rates and their relationship with packing density and flexibility. BMC Evol. Biol., 14: 78.

[26] Jack, B. R., Meyer, A. G., Echave, J., and Wilke, C. O. 2016. Functional Sites Induce LongRange Evolutionary Constraints in Enzymes. PLOS Biol., 14: e1002452.

[27] Jimenez, M. J., Arenas, M., and Bastolla, U. 2018. Substitution rates predicted by stabilityconstrained models of protein evolution are not consistent with empirical data. Mol. Biol. Evol., 35: 743-755.

[28] Joosten, R. P., te Beek, T. A. H., Krieger, E., Hekkelman, M. L., Hooft, R. W. W., Schneider, R., Sander, C., and Vriend, G. 2011. A series of PDB related databases for everyday needs. Nucleic Acids Res., 39: D411-D419.

[29] Kabsch, W. and Sander, C. 1983. Dictionary of protein secondary structure: pattern recognition of hydrogen-bonded and geometrical features. Biopolymers, 22: 2577-2637.

[30] Kimura, M. and Ota, T. 1974. On some principles governing molecular evolution. Proc Natl Acad Sci USA, 71: 2848-52.

[31] Knies, J. L., Cai, F., and Weinreich, D. M. 2017. Enzyme efficiency but not thermostability drives cefotaxime resistance evolution in tem-1 $\beta$-lactamase. Mol. Biol. Evol., 34: 1040-1054. 
[32] Liao, H., Yeh, W., Chiang, D., Jernigan, R. L., and Lustig, B. 2005. Protein sequence entropy is closely related to packing density and hydrophobicity. Protein Eng. Des. Sel., 18: 59-64.

[33] Liu, Y. and Bahar, I. 2012. Sequence evolution correlates with structural dynamics. Mol. Biol. Evol., 29: 2253-2263.

[34] López-Blanco, J. R. and Chacón, P. 2016. New generation of elastic network models. Curr. Opin. Struct. Biol., 37: 46-53.

[35] Marcos, M. L. and Echave, J. 2015. Too packed to change: Side-chain packing and site-specific substitution rates in protein evolution. PeerJ, 3: e911.

[36] Marsh, J. A. and Teichmann, S. A. 2014. Parallel dynamics and evolution: Protein conformational fluctuations and assembly reflect evolutionary changes in sequence and structure. BioEssays, 36: 209-218.

[37] Mayrose, I., Graur, D., Ben-Tal, N., and Pupko, T. 2004. Comparison of site-specific rateinference methods for protein sequences: Empirical Bayesian methods are superior. Mol. Biol. Evol., 21: 1781-1791.

[38] McCandlish, D. M. and Stoltzfus, A. 2014. Modeling evolution using the probability of fixation: history and implications. Q. Rev. Biol., 89: 225-252.

[39] McLeish, T. C. B., Rogers, T. L., Wilson, M. R., Rodgers, T. L., and Wilson, M. R. 2013. Allostery without conformation change: Modelling protein dynamics at multiple scales. arXiv.org, q-bio.BM: 56004.

[40] McLeish, T. C. B., Cann, M. J., and Rodgers, T. L. 2015. Dynamic Transmission of Protein Allostery without Structural Change: Spatial Pathways or Global Modes? Biophys. J., 109: $1240-1250$.

[41] Micheletti, C., Carloni, P., and Maritan, A. 2004. Accurate and efficient description of protein vibrational dynamics: comparing molecular dynamics and Gaussian models. Proteins, 55: $635-45$.

[42] Miller, S. R. 2017. An appraisal of the enzyme stability-activity trade-off. Evolution (N. Y)., 71: $1876-1887$.

[43] Ming, D. and Wall, M. E. 2005. Allostery in a coarse-grained model of protein dynamics. Phys. Rev. Lett., 95: 198103.

[44] Murzin, A. G., Brenner, S. E., Hubbard, T., and Chothia, C. 1995. SCOP: a structural classification of proteins database for the investigation of sequences and structures. J. Mol. 
Biol., 247: 536-540.

[45] Nelson, E. D. and Grishin, N. V. 2016. Long-Range epistasis mediated by structural change in a model of ligand binding proteins. PLoS One, 11.

[46] Nevin Gerek, Z., Kumar, S., and Banu Ozkan, S. 2013. Structural dynamics flexibility informs function and evolution at a proteome scale. Evol. Appl., 6: 423-433.

[47] Perutz, M., Kendrew, J., and Watson, H. 1965. Structure and function of haemoglobin: II. Some relations between polypeptide chain configuration and amino acid sequence. J. Mol. Biol., 13: 669-678.

[48] Ramsey, D. C., Scherrer, M. P., Zhou, T., and Wilke, C. O. 2011. The relationship between relative solvent accessibility and evolutionary rate in protein evolution. Genetics, 188: 479488.

[49] Rodrigue, N. and Lartillot, N. 2017. Detecting adaptation in protein-coding genes using a Bayesian site-heterogeneous mutation-selection codon substitution model. Mol. Biol. Evol., 34: $204-214$.

[50] Scherrer, M. P., Scherrer, M. P., Meyer, A. G., Meyer, A. G., Wilke, C. O., and Wilke, C. O. 2012. Modeling coding-sequence evolution within the context of residue solvent accessibility. BMC Evol. Biol., 12: 179.

[51] Schowen, R. L. 1978. Catalytic power and transition-state stabilization. In R. D. Gandour and R. L. Schowen, editors, Transition States of Biochemical Processes, chapter 20, pages 77-144. Springer, Boston.

[52] Shahmoradi, A. and Wilke, C. O. 2016. Dissecting the roles of local packing density and longer-range effects in protein sequence evolution. Proteins Struct. Funct. Bioinforma., 84: $841-854$.

[53] Shahmoradi, A., Sydykova, D. K., Spielman, S. J., Jackson, E. L., Dawson, E. T., Meyer, A. G., and Wilke, C. O. 2014. Predicting Evolutionary Site Variability from Structure in Viral Proteins: Buriedness, Packing, Flexibility, and Design. J. Mol. Evol., 79: 130-142.

[54] Sharir-Ivry, A. and Xia, Y. 2017. The impact of native state switching on protein sequence evolution. Mol. Biol. Evol., 34: 1378-1390.

[55] Spiess, A. N. and Neumeyer, N. 2010. An evaluation of $R^{2}$ as an inadequate measure for nonlinear models in pharmacological and biochemical research: A Monte Carlo approach. BMC Pharmacol., 10: 6. 
[56] Stein, R. L. 2011. Kinetics of Enzyme Action: Essential Principles for Drug Hunters. Wiley, Hoboken, New Jersey.

[57] Taverna, D. M. and Goldstein, R. a. 2002. Why are proteins marginally stable? Proteins Struct. Funct. Genet., 46: 105-109.

[58] Tien, M. Z., Meyer, A. G., Sydykova, D. K., Spielman, S. J., and Wilke, C. O. 2013. Maximum allowed solvent accessibilites of residues in proteins. PLoS One, 8: e80635.

[59] Torrance, J. W., Bartlett, G. J., Porter, C. T., and Thornton, J. M. 2005. Using a library of structural templates to recognise catalytic sites and explore their evolution in homologous families. J. Mol. Biol., 347: 565-581.

[60] Webb, E. C. 1992. Enzyme Nomenclature. Wiley, San Diego.

[61] Yeh, S. W., Huang, T. T., Liu, J. W., Yu, S. H., Shih, C. H., Hwang, J. K., and Echave, J. 2014a. Local packing density is the main structural determinant of the rate of protein sequence evolution at site level. Biomed. Res. Int., 2014: 572409.

[62] Yeh, S. W., Liu, J. W., Yu, S. H., Shih, C. H., Hwang, J. K., and Echave, J. 2014b. Sitespecific structural constraints on protein sequence evolutionary divergence: Local packing density versus solvent exposure. Mol. Biol. Evol., 31: 135-139. 
bioRxiv preprint doi: https://doi.org/10.1101/399154; this version posted August 23, 2018. The copyright holder for this preprint (which was not certified by peer review) is the author/funder, who has granted bioRxiv a license to display the preprint in perpetuity. It is made available under aCC-BY-NC-ND 4.0 International license.

\section{FIGURES}


A

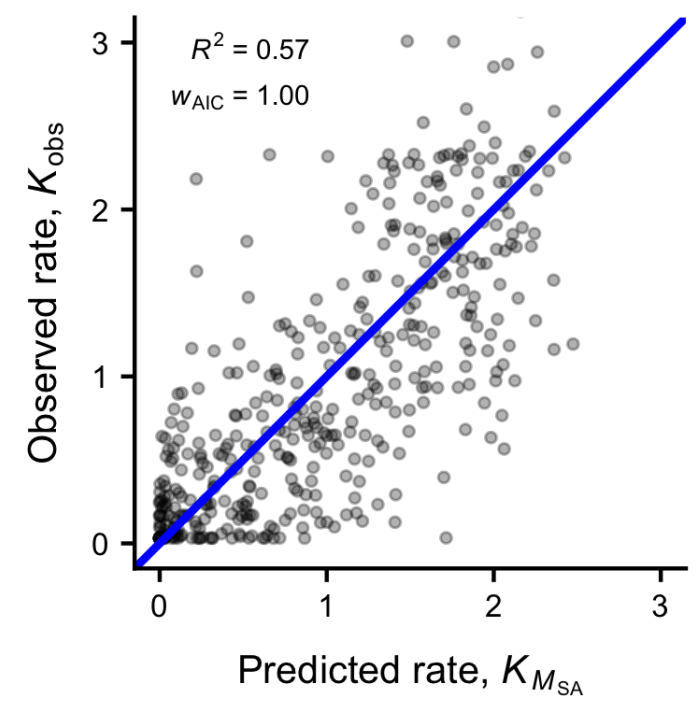

B

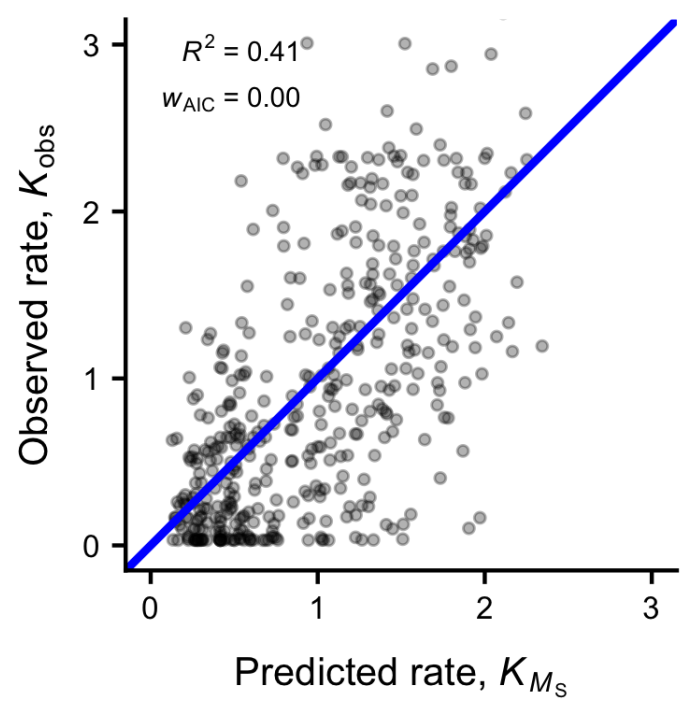

FIG. 1. Adding activity constraints improves the fit between observed and predicted rates for 1PMI. Observed site-specific substitution rates for 1PMI vs predictions of the stabilityactivity model MSA (panel A), and the stability model MS (panel B). Each point represents one protein site. Observed site-specific rates were obtained from multiple sequence alignments using the program rate4site. The square correlation coefficients are $R^{2}\left(K_{M_{\mathrm{SA}}}, K_{\mathrm{obs}}\right)=0.57$ and $R^{2}\left(K_{M_{\mathrm{S}}}, K_{\mathrm{obs}}\right)=0.41$. The best model, according to the Akaike Information Criterion, is $M_{\mathrm{SA}}$, with weight $w\left(M_{\mathrm{SA}}\right)=1.00$. 


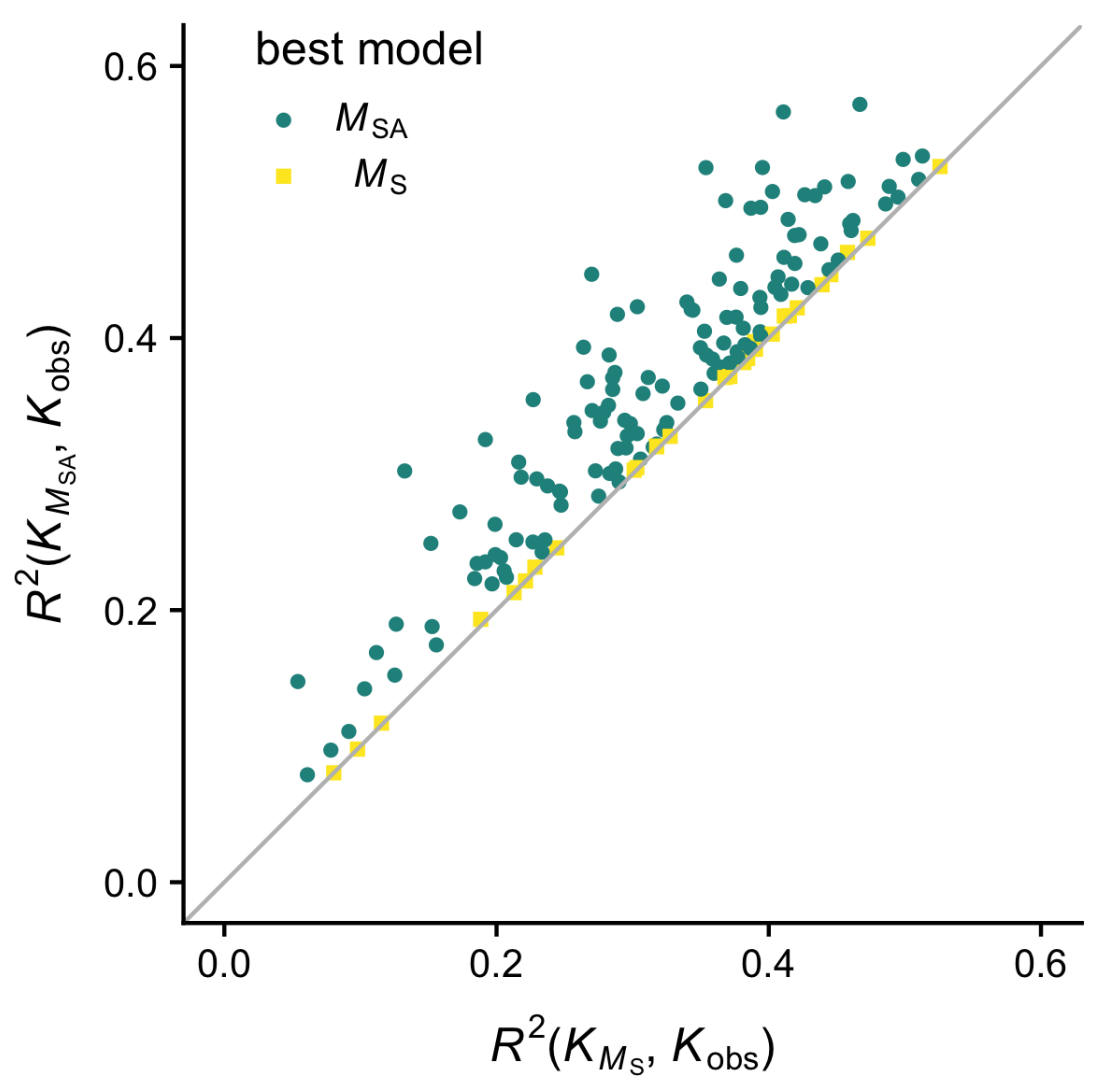

FIG. 2. MSA outperforms MS for most proteins. For each protein and each model, goodness of fit is quantified using the square observed-predicted correlation coefficient $R^{2}$ and Akaike weights. This figure shows $R^{2}$ of model $M_{\mathrm{SA}}$ vs. $R^{2}$ of model $M_{\mathrm{S}}$. The $y=x$ line is shown for reference. Colors and shapes indicate the best model according to Akaike weights (i.e. which weights is larger, $w\left(M_{\mathrm{SA}}\right)$ or $\left.w\left(M_{\mathrm{S}}\right)\right)$. For most proteins (131 out of the 160) $M_{\mathrm{SA}}$ is the best model $\left(w\left(M_{\mathrm{SA}}\right)>0.5\right)$. 


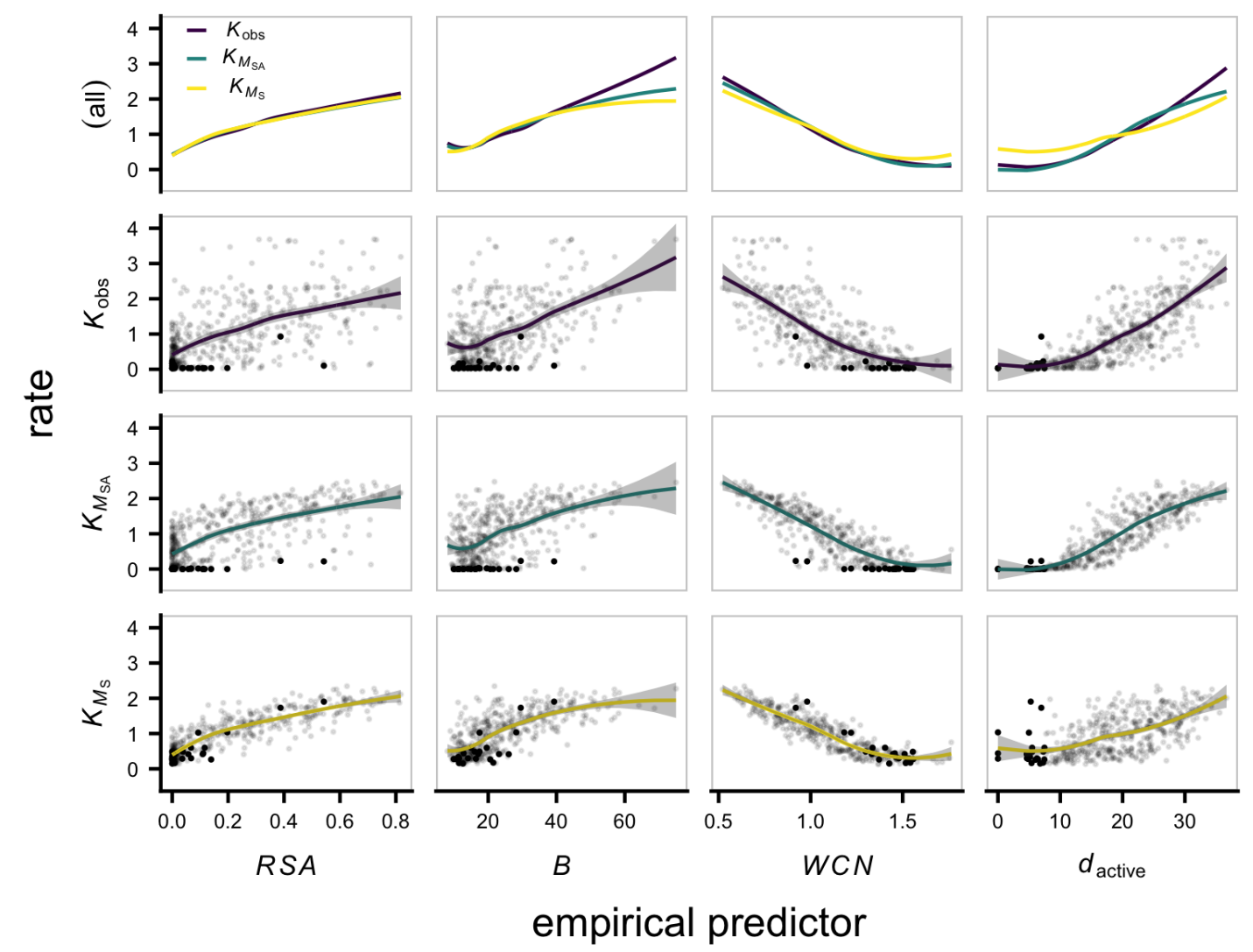

FIG. 3. $M_{\mathrm{SA}}$ provides the best fit of the dependence of site-specific rates on their structural, dynamical, and functional empirical predictors for 1PMI. This figure shows observed and predicted rates vs. four molecular traits that have been found to correlate with rates: $R S A$ (relative solvent accessibility), $B$ (X-ray B-factor), $W C N$ (weighted contact number), and $d_{\text {active }}$ (distance from closest active residue). The first row shows smooth trends, omitting sites. Rows 2-4 show the smooth fits together with actual rates for each site, highlighting active sites and their first neighbors using bolder points. While both $M_{\mathrm{S}}$ and $M_{\mathrm{SA}}$ reproduce well the observed $K-R S A, K-W C N$, and $K-B$ trends, $M_{\mathrm{SA}}$ does a somewhat better job, reproducing observed trends better and, especially, showing a similar dispersion of rates around the smooth fits (e.g. sites near the active site are far from the trend both for observed and $M_{\mathrm{SA}}$ rates, but not for the $M_{\mathrm{S}}$ case). Regarding distance to the active site, while $M_{\mathrm{S}}$ fails to reproduce the correct $K-d_{\text {active }}$ dependence, $M_{\mathrm{SA}}$ provides a good fit. Adding activity constraints is key to explain the dependence of site-specific rates on all the molecular traits that correlate with them. 


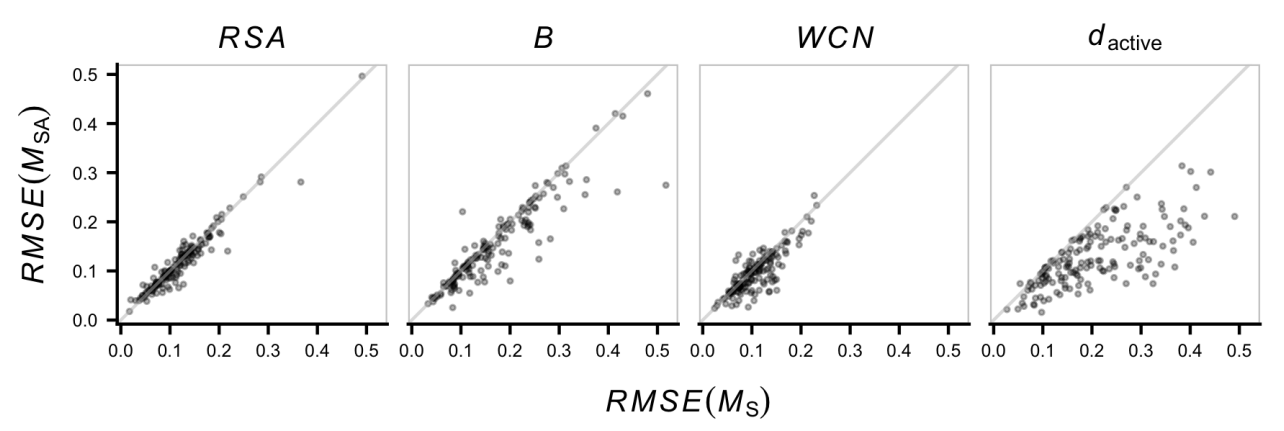

FIG. 4. MSA provide reproduces rate-predictor trends better than MS. $R M S E\left(M_{\mathrm{SA}}\right)$ $\left(\operatorname{RMSE}\left(M_{\mathrm{S}}\right)\right)$ is the Root Mean Square Error between the smooth rate-trend predicted by $M_{\mathrm{SA}}$ $\left(M_{\mathrm{S}}\right)$ and the observed trend. Each point represents one protein. RMSE quantifies the fit between predicted and observed trends (lower is better; see Materials and Methods). For points below (above) the line $y=x$, which is shown for reference, $M_{\mathrm{SA}}$ provides a better (worse) account for the trend than $M_{\mathrm{S}}$. While, both models give similar $K \sim R S A$ trends, $M_{\mathrm{SA}}$ outperforms $M_{\mathrm{S}}$ in accounting for the $K \sim W C N$ and $K \sim B$ dependences. Notably, $M_{\mathrm{SA}}$ provides a much better account of the $K \sim d_{\text {active }}$ dependence for most proteins. 
bioRxiv preprint doi: https://doi.org/10.1101/399154; this version posted August 23, 2018. The copyright holder for this preprint (which was not certified by peer review) is the author/funder, who has granted bioRxiv a license to display the preprint in perpetuity. It is made available under aCC-BY-NC-ND 4.0 International license.

\section{TABLES}


bioRxiv preprint doi: https://doi.org/10.1101/399154; this version posted August 23, 2018. The copyright holder for this preprint (which was not certified by peer review) is the author/funder, who has granted bioRxiv a license to display the preprint in perpetuity. It is made available under aCC-BY-NC-ND 4.0 International license.

\begin{tabular}{lrr}
\hline \hline trend & $n\left(M_{\mathrm{SA}}>M_{\mathrm{S}}\right) \mathrm{p}(\%) \mathrm{P}$ \\
\hline$K \sim d_{\text {active }}$ & $149 / 160$ & $932 \mathrm{e}-32$ \\
$K \sim B$ & $113 / 160$ & $719 \mathrm{e}-08$ \\
$K \sim W C N$ & $112 / 160$ & $702 \mathrm{e}-07$ \\
$K \sim R S A$ & $83 / 160$ & 520.3 \\
\hline \hline
\end{tabular}

TABLE 1. Binomial test of whether $M_{\mathrm{SA}}$ fits rate-trait trends better than $M_{\mathrm{S}} \cdot n\left(M_{\mathrm{SA}}>\right.$ $M S)$ is the number of cases for which $M_{\mathrm{SA}}$ fits observed trends better than $M_{\mathrm{S}}, p$ is the percentage of cases, $P$ is the p-value. 
bioRxiv preprint doi: https://doi.org/10.1101/399154; this version posted August 23, 2018. The copyright holder for this preprint (which was not certified by peer review) is the author/funder, who has granted bioRxiv a license to display the preprint in perpetuity. It is made available under aCC-BY-NC-ND 4.0 International license.

\begin{tabular}{lrrr}
\hline \hline trend & $<\Delta$ RMSE $>$ & t & $\mathrm{P}$ \\
\hline$K \sim d_{\text {active }}$ & -0.077 & -13.8 & 0.000 \\
$K \sim B$ & -0.017 & -5.9 & 0.000 \\
$K \sim W C N$ & -0.011 & -6.2 & 0.000 \\
$K \sim R S A$ & -0.004 & -3.0 & 0.001 \\
\hline \hline
\end{tabular}

TABLE 2. T test of whether $M_{\mathrm{SA}}$ fits rate-trait trends better than $M_{\mathbf{S}} \cdot\langle\Delta R M S E\rangle$ average over proteins of the difference between the $M_{\mathrm{SA}}$ and $M_{\mathrm{S}}$ errors of Figure. $4 . \triangle R M S E$ is negative (positive) when $M_{\mathrm{SA}}\left(M_{\mathrm{S}}\right)$ fits observed trends better. $t$ is the T-test statistic and $P$ the p-value. On average, $M_{\mathrm{SA}}$ fits all trends better than $M_{\mathrm{S}}$. 